MATEC Web of Conferences 22,03023 (2015)

DOI: $10.1051 /$ matec conf/20152203023

(C) Owned by the authors, published by EDP Sciences, 2015

\title{
Explicit-Explicit Sequence Calculation Method for the Wheel/rail Roll- ing Contact Problem Based on ANSYS/LS-DYNA
}

\author{
Hua Song, Jian Yang, Xinglong Du \& Meng Wang \\ College of Mechanical Engineering and Automation, University of Science and Technology Liaoning, Anshan, \\ Liaoning, China
}

Wei Li

China Academy of Railway Sciences, Beijing, China

\begin{abstract}
The wheel/rail rolling contact can not only lead to rail fatigue damage but also bring rail corrugation. According to the wheel/rail rolling contact problem, based on the ANSYS/LS-DYNA explicit analysis software, this paper established the finite element model of wheel/rail rolling contact in non-linear steady-state curve negotiation, and proposed the explicit-explicit sequence calculation method that can be used to solve this model. The explicit-explicit sequence calculation method uses explicit solver in calculating the rail pre-stressing force and the process of wheel/rail rolling contact. Compared with the implicit-explicit sequence calculation method that has been widely applied, the explicit-explicit sequence calculation method including similar precision in calculation with faster speed and higher efficiency, make it more applicable to solve the wheel/rail rolling contact problem of non-linear steady-state curving with a large solving model or a high non-linear degree.
\end{abstract}

Keywords: explicit-explicit sequence calculation method; wheel/rail rolling contact; ANSYS/LS-DYNA; non-linear steady-state curving

\section{INTRODUCTION}

For rails with rectilinear curve tracks, the mutual effects on the wheels and tracks are fiercer; it includes the large and ever-changing wheel/rail contact force, the crosswise creep force and its moment, and the longitudinal creep force and its moment. The repeating wheel/rail interaction of curve tracks can lead to various fatigue damage phenomena easily, and thus may affect traffic safety in a serious way. Hence scholars at home and abroad have made deep research of the wheel/rail rolling contact problem based on numerical simulation method ${ }^{[1]}$. The numerical simulation process of the wheel/rail rolling contact problem in non-linear steady-state curving is complex and time-consuming. However, a reasonable method used for solving the wheel/rail rolling contact can reduce the computing time and improve the solving efficiency to some extent.

At present, there are an appreciable quantity of documents using each solving method to calculate wheel/rail contact problems at home and abroad ${ }^{[2-6]}$ Xin Zhao et al ${ }^{[2]}$ once used explicit calculation method to analyze the process of wheel/rail rolling contact. However, they did not consider the pre-stressing force implemented on the rails at their initial positions and the initial deformation. Ping Lu et al ${ }^{[3]}$ used implicit calculation method to analyze the wheel/rail contact force. However, the time that was spent in calculating the complex three-dimensional model was too long. Xin Zhao et al ${ }^{[4]}$ analyzed the wheel-rack rolling con- tact problem of rectilinear orbit based on ANSYS/LS-DYNA implicit-explicit sequence calculation method. The results suggested that certain disturbance was made by the implicit analysis result to the transient rolling contact behavior of the explicit solving wheel set because of the existing difference between the implicit contact algorithm and the explicit contact algorithm. Besides, the disturbance degree is increased accordingly with the speeding-up of wheel velocity. Wanming Zhai et al ${ }^{[5]}$ used the Newmark explicit integration method and the prediction-correction integration method to write the program. They succeeded in accomplishing simulation for the kinematics problems of large trains in computers. This integrated method greatly improved the computing speed. However, as there're many difficulties and usage limitation in self programming, it is very unlikely to widely apply this method.

The main methods that used in the explicit analysis software ANSYS/LS-DYNA to solve wheel/rail rolling contact problems are explicit calculation method and implicit-explicit sequence calculation method. As the initial pre-stressing force of the structure needs to be considered for wheel/rail rolling contact problems, the widely-applied method is implicit-explicit sequence calculation method. It is not recommended to use the explicit calculation method as the only method because the initial pre-stressing force of the structure is not considered in this method, and it does not accord with the actual situation. However, the implicit-explicit sequence calculation method applies AN- 


\section{MATEC Web of Conferences}

SYS implicit solver to calculate the pre-stressing force and deformation of the structure which requires long computing time. In the meantime, with the increase of model freedom and non-linear degree, the computing efficiency of this method will be decreased and may lead to uncontrolled situation.

Based on the insufficiency of the current calculation method, this paper proposed the explicit-explicit sequence calculation method which has higher computing efficiency and shares similar calculation precision with implicit-explicit calculation method. The simulation results suggested that the explicit-explicit sequence calculation method is more applicable to solve the wheel/rail rolling contact problems of the non-linear steady-state curving with a large solving model and a high non-linear degree.

\section{FINITE ELEMENT MODEL OF WHEEL/RAIL ROLLING CONTACT IN NON-LINEAR STEADY-STATE CURVE NEGOTIATION}

Based on the ANSYS/LS-DYNA explicit analysis software, according to the actual wheel/rail geometrical parameters of $\mathrm{CRH} 2$ trains, the finite element model of wheel/rail rolling contact in non-linear steady-state curve negotiation is established as shown in Figure 1:

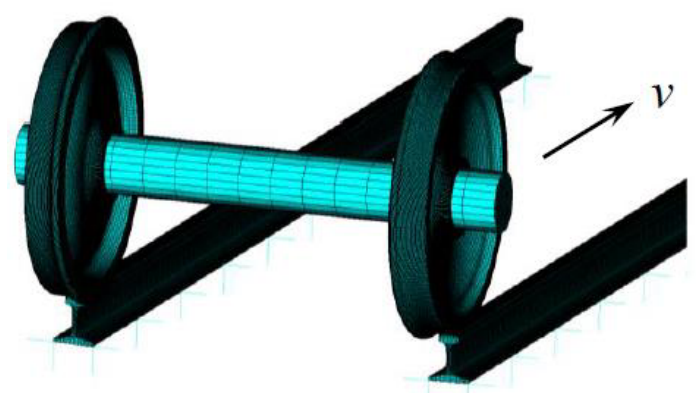

Figure 1. Wheel/rail rolling contact model of non-linear steady-state curving

The rail type is $60 \mathrm{~kg} / \mathrm{m}$ and the material type is $\mathrm{U} 75 \mathrm{~V}$. The length is $5 \mathrm{~m}$; the radius of curvature is $2000 \mathrm{~m}$; the rail cant is 1: 40 ; the diameter of wheel set is $860 \mathrm{~mm}$; and the kinematic velocity is $200 \mathrm{~km} / \mathrm{h}$. Models that made of bilinear kinematic hardening elastic plastic material are used for rails, so as to consider the plastic strain generated in the wheel/rail rolling contact process. The wheel setting of rigid body is in order to shorten the model computing time. The face-face contact algorithm shall be used for the section between wheel tracks. Hexahedral mesh divided by Solid164 entity unit is also applied. Local refinement with unit size of $0.8 \mathrm{~mm}$ shall also be implemented to the mesh of wheel/rail contact area. The total node quantity and the total unit quantity included in this model are respectively 1120830 and 1012965
The wheel/rail rolling contact finite element model of the non-linear steady-state curving uses mono-layer propping dynamic orbit model to simulate ballasted track. The kinematics model of mono-layer support track ${ }^{[6]}$ should consider the transverse and vertical stiffness damping of the track, including multiple groups of transverse springs and vertical springs. The stiffness of the vertical spring is the tandem compound of the vertical stiffness included in withhold units, ballast bed and railroad bed. However, the vertical stiffness of withhold units is equal to the sum of rectangle rubber spacer vertical stiffness and rail fastening vertical stiffness. The calculation method of mono-layer ballast bed stiffness can be used to obtain the ballast bed stiffness. The transverse spring stiffness is composed of two parts: First part is sleeper elasticity formed by the shear deformation and creeping of the concrete sleeper rail pad, while the other part is the ballast bed elasticity which is formed by the elastic displacement of ballast bed. For these two parts, constant values can be used. It can be shown in Table 1 that relevant parameters of the finite element model of wheel/rail rolling contact are in non-linear steady-state curve negotiation.

Table 1. Model Parameters

\begin{tabular}{|c|c|c|}
\hline Parameter & Parameter Setting & Value \\
\hline $\begin{array}{l}\text { Wheel/rail mate- } \\
\text { rial }\end{array}$ & $\begin{array}{l}\text { Elasticity modulus/ }(\mathrm{MPa}) \\
\text { Density/ }\left(\mathrm{kg} / \mathrm{m}^{3}\right) \\
\text { Poisson's ratio }\end{array}$ & $\begin{array}{l}2.06 \mathrm{e} 5 \\
7830 \\
0.3\end{array}$ \\
\hline $\begin{array}{l}\text { Gross mass of } \\
\text { wheel set }\end{array}$ & $\begin{array}{l}\text { Wheel set quality/ }(\mathrm{kg}) \\
\text { Axle load/ }(\mathrm{kg})\end{array}$ & $\begin{array}{l}580 \\
16000\end{array}$ \\
\hline Clip fastening & Vertical stiffness $(M N / m)$ & 3.85 \\
\hline $\begin{array}{l}\text { Rectangle rubber } \\
\text { spacer }\end{array}$ & $\begin{array}{cc}\text { Vertical stiffness } & (M N / m) \\
\text { Transverse } & \text { stiffness } \\
(M N / m) & \end{array}$ & $\begin{array}{l}80 \\
7\end{array}$ \\
\hline Ballast bed & $\begin{array}{cc}\text { Vertical stiffness } & (M N / m) \\
\text { Transverse } & \text { stiffness } \\
(M N / m) & \end{array}$ & $\begin{array}{l}220 \\
0.2\end{array}$ \\
\hline Railroad bed & Vertical stiffness $(M N / m)$ & 130 \\
\hline Vertical spring & $\begin{array}{l}\text { Stiffness/ }(M N / m) \\
\text { Damping/ }(k N \cdot s / m)\end{array}$ & $\begin{array}{l}42.63 \\
139.8\end{array}$ \\
\hline $\begin{array}{l}\text { Transverse } \\
\text { spring }\end{array}$ & $\begin{array}{l}\text { Stiffness/ }(M N / m) \\
\text { Damping/ }(k N \cdot s / m)\end{array}$ & $\begin{array}{l}7.2 \\
108.8\end{array}$ \\
\hline
\end{tabular}

3 CHARACTERISTIC ANALYSES OF THE ANSYS/LS-DYNA EXPLICIT ALGORITHM AND THE ANSYS IMPLICIT ALGORITHM

The ANSYS/LS-DYNA explicit algorithm applies the central difference method that replaces differentials with finite differences and expresses the first and sec- 
ond derivatives (namely velocity and acceleration) of displacement by means of linear extrapolation. There is no need to complete the equilibrium iteration or calculate the unit tangent line matrix. No approximation problem exists in this method. Compared with the ANSYS implicit algorithm, this method requires less internal storage. Although the central difference method needs certain conditional stability, it is widely used to solve high-speed transient problems due to its high computing efficiency.

The ANSYS implicit algorithm applies the Newmark method based on virtual work principle. No inertial effect is considered in this method. The iteration solving process is needed within each increment step for static equilibrium equation. As this method is highly precise and the iteration solving process is needed to solve large-scale linear equation sets each time, it is very applicable for calculating static problems. However, it occupies a significant quantity of computing resource, disk space and internal storage, leading to the low computing efficiency. For linear problems, the ANSYS implicit algorithm has no conditional convergence and it can set the big time step. Nevertheless, for non-linear problems such as wheel/rail rolling contact, very tiny time steps need to be set up in order to ensure convergence, resulting in multiplied calculation time that may seriously affect computing efficiency.

The calculation time of ANSYS/LS-DYNA explicit algorithm is in direct proportion to the node number of model and in inverse proportion to the minimum unit dimension of model. Meanwhile, more experience has showed that the calculation time required in implicit algorithm is generally in direct proportion to the square of model freedom degree (in direct proportion to number of node). As a result, if the dimension of the mesh stays the same, with the increase of model specification and number of node, the calculation time required in the ANSYS/LS DYNA explicit algorithm will be less than that of ANSYS implicit algorithm and the computing efficiency of ANSYS/LS-DYNA explicit algorithm will be higher. Moreover, by sacrificing certain computing precision, the ANSYS/LS-DYNA explicit algorithm can replace the ANSYS implicit algorithm to solve statics problems and apply pre-stressing force and deformation to the rails, so as to reduce the computing time. The calculation process of wheel/rail rolling contact includes two parts: the process of applying initial pre-stressing force and initial deformation to the rails and the process of computing wheel/rail rolling contact. The application of initial pre-stressing force and initial deformation to the rails aims at accelerating the stable wheel velocity. The minor computational error made in initial pre-stressing force and initial deformation calculation will leave little impact on the stable velocity of wheel/rail operation. Therefore, this paper tried to use the explicit-explicit sequence calculation method to calculate the wheel/rail rolling contact problem of non-linear steady-state curving.

\section{EXPLICIT-EXPLICIT SEQUENCE CALCULA-} TION METHOD

\subsection{Implementation steps of explicit-explicit sequence calculation method}

The computing process of explicit-explicit sequence calculation method is composed of three parts: In the situation that axle load is only applied to wheel sets, it is used to calculate the pre-stressing force and initial deformation applied to the model at the initial position under corresponding boundary conditions; import the computing result of the last sub-step (wheel sets are basically in static state at the moment, the result is similar to that of ANSYS implicit algorithm) into the finite element model and update the initial pre-stressing force and deformation of the rails; apply heavy loading of axle load, transverse centrifugal force and corresponding boundary conditions to wheel sets and use their initial velocity to calculate the process of wheel/rail rolling contact. The specific boundary conditions shall be set as follows: apply symmetric boundary conditions and the external end nodes of full-constrained transverse springs and vertical springs on the ends of the two rails.

\subsection{Contrastive analysis of explicit-explicit sequence calculation method and implicit-explicit sequence} calculation method

Figure 2 is shown for the comparison of explicit-explicit sequence calculation method and implicit-explicit sequence calculation method. From Figure 2, it can be easily seen that the differences existing between these two methods mainly lie in the calculation process of initial pre-stressing force and deformation applied to rails, and thus the differences will bring certain relative deviation in the computing results. The calculation of initial pre-stressing force and deformation applied to rails is a typical process of statics analysis. During the calculation process of $\mathrm{AN}$ SYS/LS-DYNA explicit algorithm, inertial effect needs to be considered. However, due to its static equilibrium principle, the ANSYS implicit algorithm does not consider any inertial effect. The inertial effect of wheel sets will bring certain impact on steel rails, thus make the computing results of the initial pre-stressing force and deformation of the rails obtained by the ANSYS/LS-DYNA explicit algorithm slightly bigger than that obtained by ANSYS implicit algorithm. Certain numerical fault will be generated accordingly. Figure 3 is shown for the vertical displacement distribution of ANSYS/LS-DYNA explicit solution and ANSYS implicit solution along the vertical axis of contact spots in the initial contact area. From Figure 3, it can be seen that according to the calculation results of the initial pre-stressing force and deformation applied to rails, the error made in ANSYS/LS-DYNA explicit solution is slightly smaller than that of ANSYS implicit solution. Furthermore, the error made in ANSYS/LS-DYNA explicit solution can be reduced by increasing the damping beneath the 
MATEC Web of Conferences

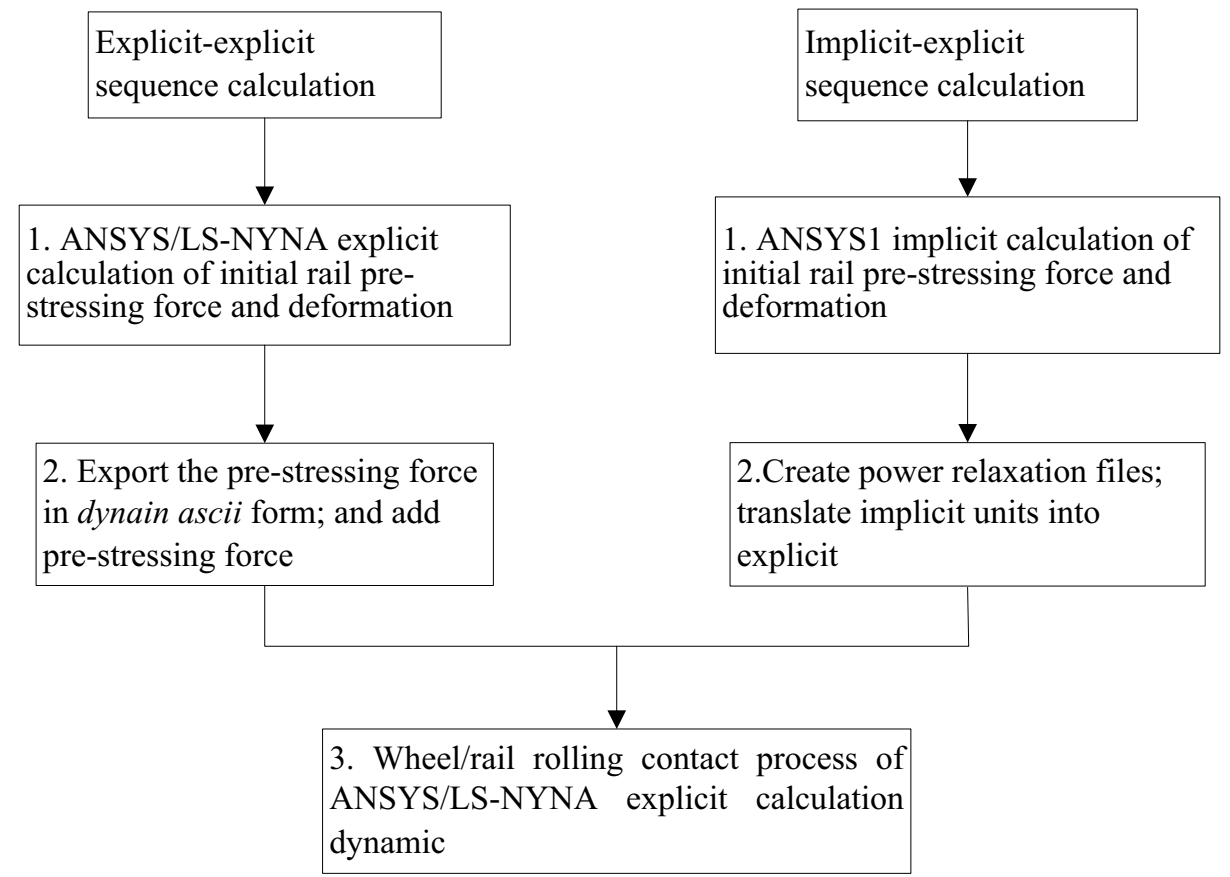

Figure 2. Comparison of explicit-explicit sequence calculation method and implicit-explicit sequence

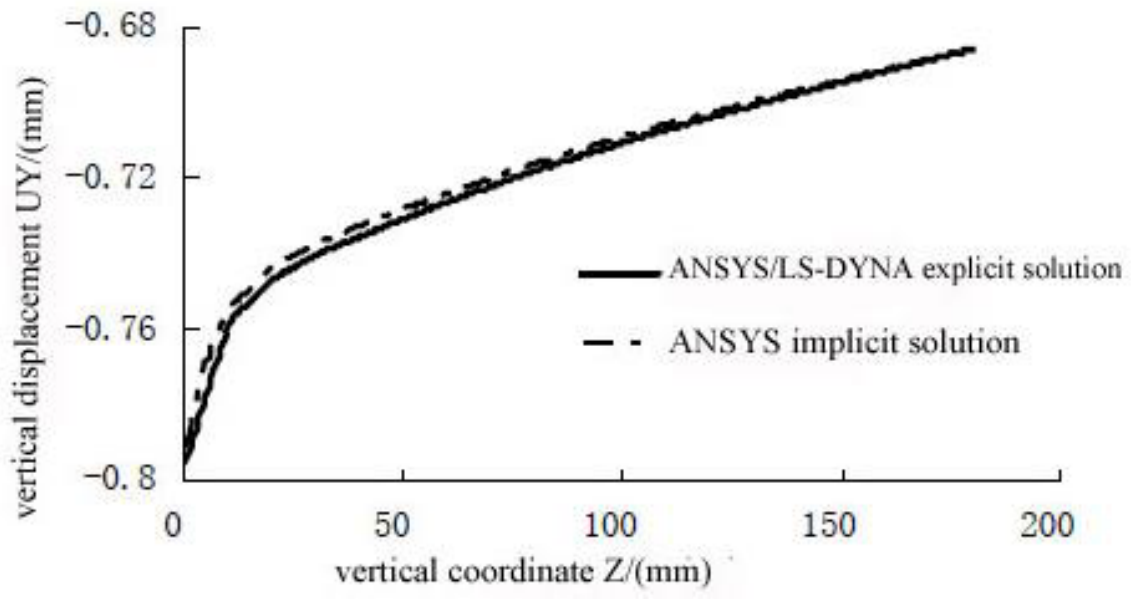

Figure 3. Vertical displacement distribution curve of explicit calculation and implicit calculation

rails or slightly extending the calculation time.

The time history curve of wheel-set vertical velocity within $0 \sim 0.04 \mathrm{~s}$ is shown in Figure 4 . From this figure, it can be seen that due to the effect of the damping on sub-rail foundation, the vertical velocity of wheel sets will be in gradual attenuation and become stable at around $0 \mathrm{~mm} / \mathrm{s}$ on the time point of $0.04 \mathrm{~s}$ which can suggest that the wheel sets are close to be static. By this time, the computing precision of ANSYS/LS-DYNA explicit solution and ANSYS implicit solution are basically the same. Meanwhile, after using these two methods to solve the finite element model of non-linear wheel/rail rolling contact respectively, the results can show that the calculation time used in the ANSYS/LS-DYNA explicit algorithm is about $1 / 4$ of that used in the ANSYS implicit algorithm (which is based on Intel(R) Core(TM) i7-3930K CPU @ $3.20 \mathrm{GHz}, 16 \mathrm{G}$ RAM). It can be seen that ANSYS/LS-DYNA has higher computing efficiency in this situation. 
ICETA 2015

As the processes of solving dynamic wheel/rail rolling contact on the third step are basically the same in the explicit-explicit sequence calculation method and the implicit-explicit sequence calculation method, it can be concluded that the explicit-explicit sequence calculation method shares the same computing precision with implicit-explicit sequence calculation method. However, compared with the latter one, the explicit-explicit sequence calculation method has higher computing efficiency that makes it more applicable for solving the wheel/rail rolling contact problem of non-linear steady-state curving with a large solution model or with a high non-linear degree.

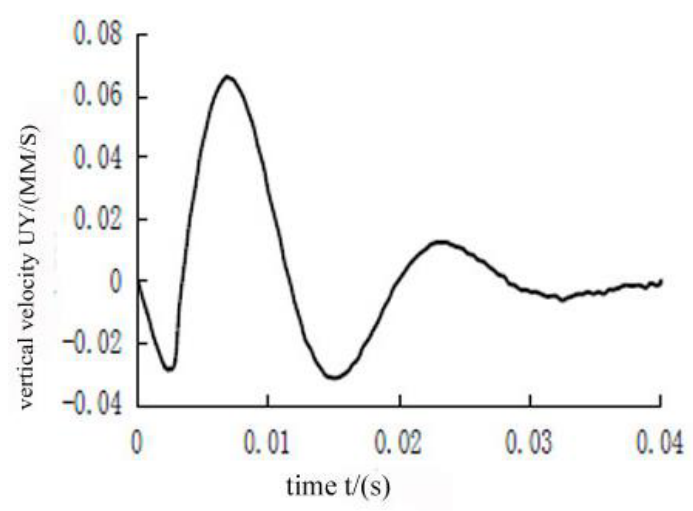

\section{CONCLUSION}

This paper established the finite element model of wheel/rail rolling contact in non-linear steady-state curve negotiation based on the explicit analysis software ANSYS/LS-DYNA and proposed the explicit-explicit sequence calculation method used for solving this model. By calculating the non-linear wheel/rail rolling contact finite element model, it can be concluded that compared with the implicit-explicit sequence calculation method, the explicit-explicit sequence calculation method basically shares the same calculation error. However, the calculation efficiency of explicit-explicit sequence calculation method is higher, and thus makes it more applicable for solving the wheel/rail rolling contact problem of non-linear steady-state curving with a large solution model or with a high non-linear degree.

\section{REFERENCES}

[1] Jin X. S. \& Shen Z.Y. 2001.The Latest Development of the Study on Wheel/Rail Rolling Contact Fatigue Problems. Journal of Railway. 02: 92-108.

[2] Xin Zhao, Zefeng Wen \& Minhao Zhu, eta. 2014. A Study on High-speed Rolling Contact between a Wheel and a Contaminated Rail. Vehicle System Dynamics, 52(10): 1270-1287.
[3] Lu P. Cui D.B. \& Wang N. 2009. The Numerical Calculation Methods of Contact Forces between Wheel and Track. Lubrication Engineering. 07: 28-32.

[4] Zhao X., Wen Z.F. \& Wang H.Y., et al 2013.The Finite Element Model of Three-Dimensional High-Speed Wheel/rail Transient-State Contact and Its Application. Journal of Mechanical Engineering. 49(18): 1-7.

[5] Di W.M. \& Huang Z.H. 1991. Analysis Methods of the Non-Linear Numerical Values of Train Dynamics. Journal of Southwest Jiaotong University. 33(01): 82-90.

[6] Chang C, Wang C. \& Chen B, et al. 2010. A Study of a Numerical Analysis Method for the Wheel-Rail Wear of a Heavy-Haul Train. Proc IMechE, Part F: J Rail and Rapid Transit. 224(5): 473-482.

[7] Di W.M. 2007. Vehicle-Track Coupling Dynamics (3rd Edition). Beijing: Science Press.

[8] Lian S.L. 2004. Theoretical Calculation of Track Structural Stiffness. China Railway Science. 25 (01): 68-72. 